\title{
Research On a Real-time Database of General Engineering Flight Simulation
}

\author{
Cong Zhang \\ School of Computer Science \& Engineering \\ Beihang University \\ Beijing, China \\ e-mail: zhangcongcong0823@163.com
}

Hong Xu

School of Computer Science \& Engineering

\author{
Beihang University \\ Beijing, China \\ xh@buaa.com
}

Weiqun Shen

School of Automation Science and Electrical Engineering

Beihang University

Beijing, China

e-mail: shenweiqun@buaa.com

\begin{abstract}
General Engineering Flight Simulation Database is a important subsystem of Engineering Flight Simulation Platform. Engineering Flight Simulation Platform, which is oriented to the development of new aircraft, is a indispensable equipment for developing new aircraft, researching flight theory and flight control. During the simulation, engineering flight simulation system will produce a large real-time simulation data. The researchers discover the problems during the development of new aircraft, through the flight simulation database's processing of data. This article discusses using XML files as data conversion intermediate medium, dynamic method of building a data table in a database, and fast storage, fast retrieval methods of the huge amount of data in real-time simulation .The practice shows that the application of these methods are applied for processing a variety of flight simulation data fast.
\end{abstract}

Keywords: database; flight simulation platform; xml

\section{INTRODUCTION}

Engineering Flight simulation platform is a kind of flight simulation system, which is research-oriented. It is a indispensable equipment when a new aircraft is developed, theory of flight and flight control are researched. It brings together simulation platform management, flight test, test data analysis, test information management and many other functions. In addition, it provides a paperless new aircraft design management platform for the aircraft designer, which is good for security management. In summary, it has high economic value and military value.

In order to analyze the simulation process and to improve the design of the aircraft, the engineering flight simulation platform needs to store the real-time data in simulation process. And in the simulation process, it is needed to store several thousand bytes of data. Therefore, it is not store those data to the database during the simulation process[1]. In order to store these real-time data, one of the methods is to store the data directly to a data file firstly.
After the end of the simulation, format these data in data files and then insert them to the corresponding data table. These operations make it convenient to search and analyze the simulation data for researchers.

How to make a database quickly handle all types of different models and different experimental simulation data, is the key issue of this thesis.

\section{THE FUNCTION AND STRUCTURE OF THE DATABASE}

The database mentioned in this paper, is based on relational database, and is one part of the engineering flight simulation platform which is used to process data. It uses the adding, deleting, modifying and searching function which is provided by the underlying database, to realize its function of analyzing, storing and post-processing the binary package[2].

\section{A. The function of the database}

- Dynamic building and importing to a data table functions

Select parameter variables which the researchers are concerned with, from the parameter database. Then create a $\mathrm{xml}$ file using the selected variables. Analyze the xml file. According to the analytical results, build a data table in the database dynamically. Also in accordance with the analytical results of xml file, parse the binary data, and then put the analytical data into the new table that we build just now.

- Viewing and searching data functions

As to the data which has been stored in database, when the researchers need to view the data, it can be fetched and display on the screen. And if the researchers want to view a part of the data they concern with, it also can search and display the data according to the request which is input by the researchers.

- Maintenance function

The data table in database and the binary compressed files can be deleted, if they are not needed at all.

- Post-processing functions 
After laying in the data in database, in order to observe the changes and the overall situation of the data directviewingly and conveniently, it can draw curves using the specified data.

- $\quad$ Saving function

You can export the data from the database, and then save them as a text file.

\section{B. The structure of the database}

The structure model of database is shown in Figure 1:

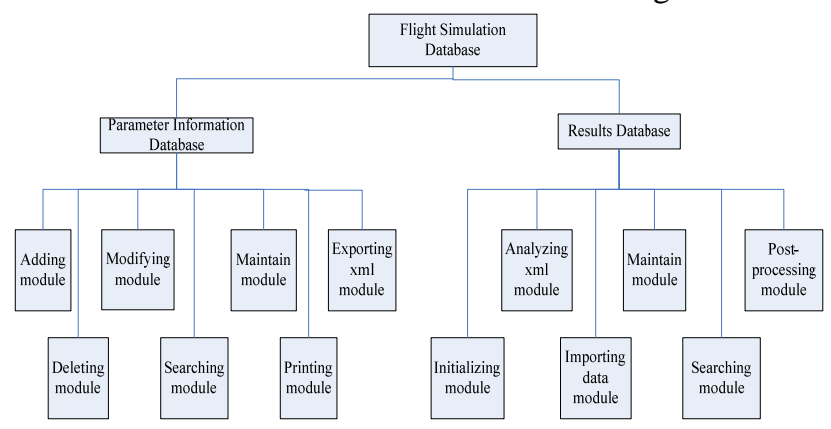

Figure 1 structure of database model diagram

Database is divided into two parts, the parameter information database and the results database.

Parameter information database is used to store all the flight parameter information, the researchers could add, modify, delete, query, print parameter information, select the parameters and generate an XML file which is used by results database. Create a data table dynamically by selecting variables that the researchers are interested in.

Researchers associate XML files with the result database, the program dynamically create data tables by parsing an XML document, and through analyzing the xml file obtain the corresponding experimental data from binary compressed files stored by other programs from the simulation system. Then store the data to underlying database, prepare to postprocess.

\section{BUILDING LIBRARY DYNAMICALLY}

\section{A. Building library dynamically}

$\mathrm{Xml}$, that is the eXtensible Markup Language. It can be used as a standard interchange language, and be responsible for describing the exchanging data[3]. It has excellent interoperability and reliability. Furthermore, it has a property that can separate data from its presentation. And it also has powerful data expression ability.

It developed as a symbolic markup language based on the HTML. It has the following advantages: (1) Separate Data from its representation ; (2) It is a non-markup language, researchers can define their own DTD; (3) Its main contents is the data; (4) It is able to encapsulate the data source, so as to mask the data source. Through the xml's integration, it achieve data exchange and sharing between different data sources.
Document Object Model (DOM) is an important part of the XML, which defines the way the process executing the document, uses a tree structure to present a xml document, and allows the researchers to access the information in the DOM. Various kinds of computer languages offer a wide interface to it, And it is widely used.

The above mentioned features of xml determine its important position in the transmission of information in today's society. And these features are also the basis and key technique for building a data table in a database dynamically.

XML files are throughout the system. Each flight parameter, are recorded by an XML file. The data model is shown in Figure 2.

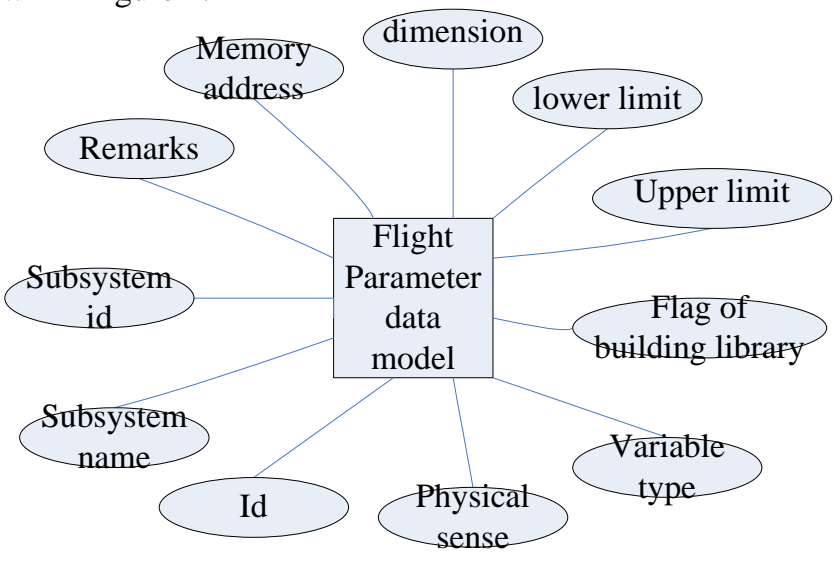

Figure 2 flight parameter data model

Refer to the data model for the flight parameters, we can define the xml file format as follows:

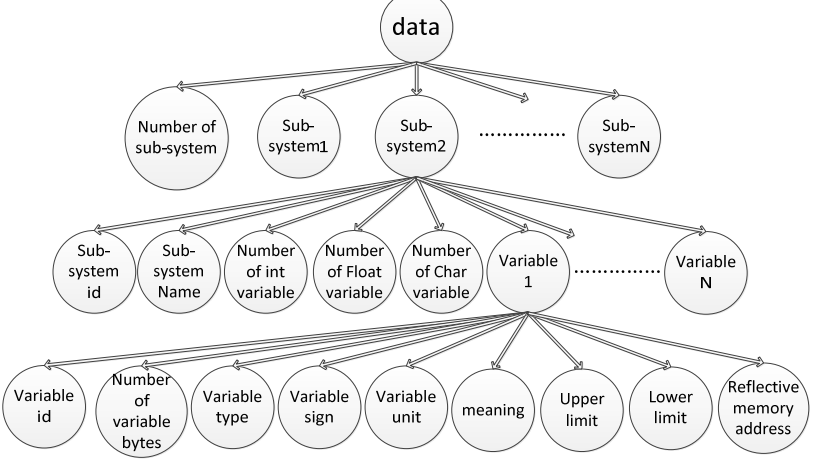

Figure $3 \mathrm{xml}$ file storage format

According to the storage structure shown in Figure 3, in a simulation process, save the data that belongs to the same sub-system as a single file. Each data occupies double words in each data file.

In practical applications, to complete a simulation test, the engineering flight simulation system usually need to define a number of parameters (the system has thousands). However, during the process of analyzing the simulation results, if the researchers want to view all the data, there are some inconveniences. Firstly, in the several thousands of variables, finding the values of some variables which the researchers are concerned about is so inconvenient and easy 
to make mistakes. Most time must be wasted to distinguish whether it is the data I want or not.

In addition, importing the data to the database and Exporting the data from the database will consume a lot of time and resources. But if we conduct an experiment only store one part of the total variables, when we want to view one or several variables which are not stored for some reasons. At this time, it will lead to greater human and material waste.

For all of these, the system uses the recording of all test data and compressed (saved as a binary file, the compression rate of up to 200 times), the results of the researchers focus only on their own building a database parameter data methods to solve this problem.

The selection whether choose the variable or not depends on "building table's flag" item in parameter information mentioned in Figure 2. Value of this parameter could be 0 or 1 . If the value of parameter is 1 , it means that we will contain it in the xml file. But if the value is 0 , that is to say we will not use it. After the selection, we will generate an XML file for the result database to dynamically create data table. “CPS_A4_format”.

\section{B. The design of data format}

The Engineering Flight Simulation Platform is a distributed system. Each simulation cycle, each subsystem completes their simulation task, each subsystem contains a large number of variables, each variable with its own different properties[4]. For completing to analyze the large amounts of data generated in the simulation process, a good design of the data structure is very important.

Refer to the store structure showed in Figure 2, the data structure can be designed as follows. There are some little changes with the structure mentioned in Figure 2. It can be more convenient when we deal with the history data after these changes.

Data Structure $::=<$ total number of subsystems, the total number of variables $>$

Subsystem node :: = <subsystem number, subsystem name, the number of variables, all variable information>

Variable Information :: = <ariable number, variable type, variable name, variable dimensions, physical sense, discrete markers, discrete quantum number of variables, the sub-variable information>

Sub-variable information :: = <sub variable name, the start bit, stop bits>

Reflective Memory address is required when storing data in real-time. As to historical data processing, it is not required. It can be omitted. Since the engineering flight simulation platform is a typical semi-physical simulation system, communication between various systems in addition to the commonly used floating-point data, there are many non-standard data, which comes from different data sources. Such as character data, each of one or a combination of each expresses several different meanings. So there needs to determine whether it is discrete, the number of discrete variables, and the sub-variable information.

\section{Data analysis}

After being acquaintance with data's content and formation, the work of data analysis follows.

The first step of data analysis is to find all the variables in the simulation recorded in the XML files ${ }^{[5]}$, from which information of all variables can be obtained and new XML files recording variables we concerned can be generated. Obtaining the information of variables to be analyzed is a procedure of resolving XML files and loading subsystem names, variable names and other information recorded in the $\mathrm{XML}$ files to the data structure designed in the previous chapter.

From the subsystem names, corresponding files can be found and the variable number is available for obtaining the position of each variable in every millisecond.

\section{Data import to the database}

The template is used to format your paper and style the text. All margins, column widths, line spaces, and text fonts are prescribed; please do not alter them. You may note peculiarities. For example, the head margin in this template measures proportionately more than is customary. This measurement and others are deliberate, using specifications that anticipate your paper as one part of the entire proceedings, and not as an independent document. Please do not revise any of the current designations.

The data structure in binary files is shown in figure 4

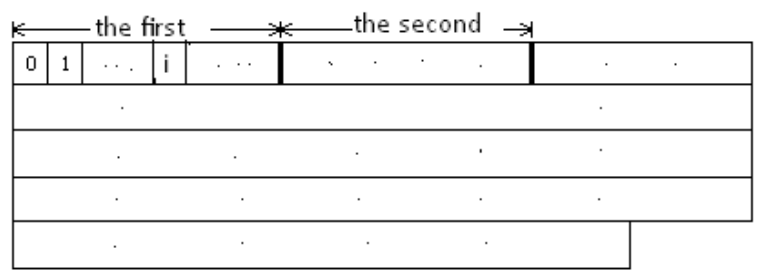

Figure 4 the model of the binary file

Based on established database, the function of this part mainly focus on reading corresponding data from binary files generated by other program, converting the data to decimal number and recording it to temporary intermediate files. The structure of binary files is shown in figure 4, where every group of data, separated by thick lines, indicates each variable value in different time.

Taking importing the ith data value in the Nth subsystem where the total variable quantity is $\mathrm{N}$ as example, we read $\mathrm{N}$ binary numbers to array Value[MAX] and convert Value[i] to decimal number to be written into intermediate files. After processing all the variables with such procedure, the data can be imported to database through SQL statements below:

LOAD DATA INFILE [the complete intermediate file's path] INTO TABLE [table name just created dynamically] FIELDS TERMINATED BY ','

Now researchers can analyze data in simulation.

Only by researcher's generating sub-XML files recording variables concerned and relating them with corresponding data files to establish database, the program can fulfill the 
work of obtaining data for analysis from binary files. The function of dynamic database establishment guarantees the system's automation and eliminates the restriction of data's source, which implement the sharing of data from different origin.

\section{FAST RETRIEVAL OF DATA}

After importing data to database, researchers can analyze the data to improve the airplane design. The retrieval function is necessary for huge data amount.

\section{A. The Relationship Between Field Numbers And Retrieval Speed}

Since a lot of functions such as resolving XML files and initializing the data structure designed in chapter 1 from XML files have been implemented, we can describe variables to be obtained with subsystem names and variables' physical meanings. Researchers can easily retrieve data by giving the scale in some aspects.

But increasing the speed of retrieval has always been a important task because the process of huge amount data retrieval is very slow.

We assume that the field numbers bear some links with the retrieval speed. After some experiments, the relationship between them is depicted in figure 5 .

Figure 5 indicates that with the increase of field numbers, the retrieval speed decreases. Hence, reducing the quantity of field numbers can be a effective way.

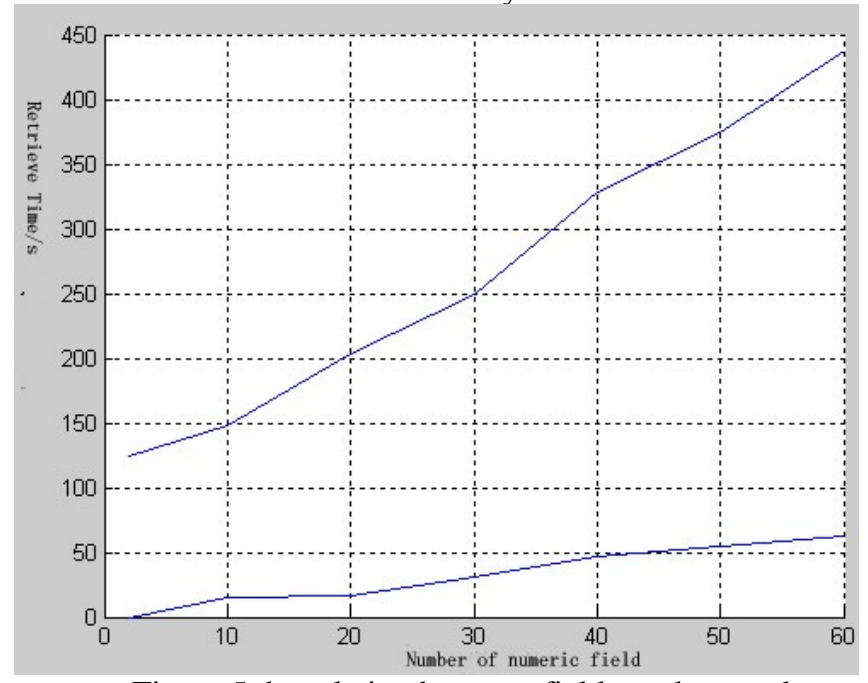

Figure 5 the relation between field numbers and retrieving speed

\section{B. The relationship between index numbers and retrieval} speed.

Another way to increase retrieval speed is to establish index.

To improve the index efficiency, principles such as which field to be based on and which type of index to be established should be taken into consideration.

Priciples listed below can be adopted to establish an index:

- Use a zero before decimal points: “ 0.25 ”, not “.25”.
- Choose unique index. The value of unique index must be unique, which means the process of searching a certain record can be faster.

- Establish index for fields frequently facing sorting, grouping, uniting and enquiry.

- Limit the amount of index. Index will occupy the room of harddisk.

- $\quad$ Limit the number of index.

If the number of the index is too large, the speed you search may be slower. Each index needs disk space, the more the index is, the larger the space is occupid. When we modify the tables, it is so troublesome to update and reconfigure。The more the index is, the slower you update the data table. It may waste lot of time.

- Delete the index which is not used any more or is used rarely.

Follow the above principles, at the same time of creating data table, add an appropriate index, and it is also a way to improve retrieval efficiency. But as described above, the establishment of the index is not unlimited. If the number of index is too large, it will also slow the retrieval speed.

According to these principles, through experiments, the result is as shown in figure 6. Figure 6 is the result based on a 10 fields' table.

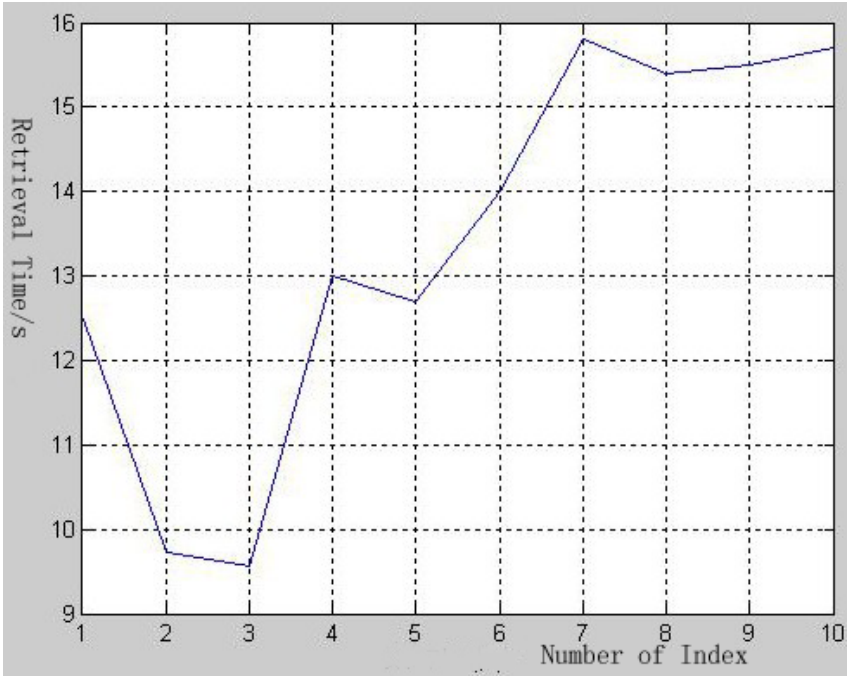

Figure 6 the relation between index numbers and retrieving speed

\section{Method to create index}

When building library dynamically, attention should be paid to make the number of fields fewer in a table as far as possible[5]. And at the same time create index in the table in order to speed the data retrievaling, and it is important to determine the number of index according to the number of the fields in table. eg. ten fields, you can set up two or three index is more appropriate. 


\section{CONCLUSION}

The General Engineering Flight Simulation real-time database this paper shudied has been successfully used in a flight simulation platform. The application practice shows that this database can meet the researchers for rapid processing and analysis of all kinds of real-time data in engineering flight simulation. And it supports the new research in out country greatly.

\section{REFERENCES}

[1] M D G. Browse Journals \& Magazines > Software, IEEE ...> Volume:22 Issue:3 MySQL: lessons learned on a digital library[C]. Software, IEEE, 2005:10 - 13.

[2] Dandan F, Tao L. Study of A Dynamic Database Modeling Based on XML In Engineering Flight Simulator[J]. COMPUTER SYSTEMS \& APPLICATIONS, 2007(12).

[3] Chao W. The Implementation Research on The Index Structure of Database[D]. Tianjin:Tianjin University, 2006.

[4] Xiajun J, Huizhong W, Weiqing L. Research of Real-time Database Technology in Data Collection of Simulation[J]. JOURNAL OF SYSTEM SIMULATION, 2005, 17(8):1872-1874.

[5] Lijun X, Jun Y. Comparison of Several Indexes[J]. JOURNAL OF HUAIHUA UNIVERSITY, 2009, 28(8). 